DOI: $10.15593 / 2224-9982 / 2016.44 .06$

УДК 534-13

\author{
Л.Н. Бутымова, В.Я. Модорский, Н.А. Шевелев
}

Пермский национальный исследовательский

политехнический университет, Пермь, Россия

\title{
ЧИСЛЕННОЕ МОДЕЛИРОВАНИЕ
}

\section{ГАЗОДИНАМИЧЕСКИХ ПРОЦЕССОВ В ЭНЕРГЕТИЧЕСКОЙ УСТАНОВКЕ НА БАЗЕ ДВИГАТЕЛЯ СТИРЛИНГА}

\begin{abstract}
Разработаны фризическая модель и расчетная схема, в соответствии с которыми модельная энергетическая установка на базе двигателя Стирлинга описывается двумя подвижными элементами в замкнутом объеме. Задача решается в связанной однонаправленной нестационарной численно-аналитической 3D-постановке. Оба элемента совершают возвратнопоступательные движения по заданному закону. Учитывается запаздывание одного элемента относительно другого. В ходе газодинамического расчета определяются газодинамические силы, действующие на подвижные элементы. Вывод об эффективности рабочего процесса в двигателе Стирлинга делается по результатам анализа сдвига фаз колебаний подвижных элементов и газодинамических сил, действующих на них. На основе вычислительного эксперимента проведен анализ соответствия колебаний подвижных элементов и газодинамических сил. Выявлены зависимости колебаний газодинамической силы, действующей на поршни, от перемещений поршней, сдвига фраз колебаний, наличия подвода тепла. Выявлены зависимости сдвига фаз колебаний между поршнем и действующей на него газодинамической силой $\left(\varphi_{2}, \varphi_{3}\right)$ от сдвига фаз колебаний поршней $\left(\varphi_{1}\right)$. Однозначной взаимосвязи колебаний газодинамической силы и соответствующих им перемещений поршней не обнаружено. Результаты получены в системе инженерного анализа ANSYS CFX, с использованием высокопроизводительного вычислительного комплекса Пермского национального исследовательского политехнического университета.

Ключевые слова: двигатель Стирлинга, численное моделирование, ANSYS CFX, вычислительный эксперимент, газодинамика, колебания, сдвиг фаз колебаний, вибрации, физическая модель, переходные процессы, аэроупругость.
\end{abstract}

\section{L.N. Butymova, V.Ya. Modorskiy, N.A. Shevelev}

Perm National Research Politechnic University, Perm, Russian Federation

\section{NUMERICAL MODELING OF GAS DYNAMIC PROCESSES IN POWER EQUIPMENT BASED ON STIRLING ENGINES}

Model power equipment based on Stirling engine described by two movable elements in the closed volume is developed. For the model of the power equipment is designed physical model and the calculation scheme. The task is solved in a coupled unidirectional unsteady numerical - analytical 3D formulation. Both elements make a reciprocating motion by a given law. Delay of one element relative to the other is taken into account. The gas-dynamic forces acting on the movable elements are determined 
in the gas-dynamic calculation. The conclusion about the effectiveness of the working process in the Stirling engine is based on analysis of the phase shift of oscillations of movable elements and gasdynamic forces acting on them. Based on computing experiment the analysis of conformity between the oscillations of moving elements and gasdynamic forces is carried out. It is obtained the dependence of oscillations of the gas-dynamic forces on the piston displacement, the shear oscillation phase and heat input. Dependences of the phase shift between the oscillations of the piston and gas-dynamic force acting on it $\left(\varphi_{2}, \varphi_{3}\right)$ on the phase shift of the oscillation of pistons $\left(\varphi_{1}\right)$ are revealed. The one-to-one correspondence of the oscillations of gas dynamic forces and their corresponding pistons displacement escaped detection. The results are obtained by the system engineering analysis ANSYS CFX, using highperformance computing system of the Perm National Research Polytechnic University.

Keywords: Stirling engine, numerical modeling, ANSYS CFX, numerical simulation, gas dynamics, oscillations, oscillation phase shift, vibrations, physical model, transient processes, aeroelasticity.

\section{Введение}

Одним из возможных путей совершенствования энергетических установок (ЭУ) летательных аппаратов является применение двигателя Стирлинга. К преимуществам двигателя Стирлинга можно отнести высокий КПД, бесшумность, высокий моторесурс, возможность использования различных топлив и т.д. Конструкция двигателей Стирлинга отличается простотой, вместе с тем в них протекают сложные процессы, требующие учета пространственной неравномерности потока, тепловых потоков, охлаждения и т.д.

Рассмотрим процессы, происходящие в двигателе Стирлинга. Двигатель Стирлинга - это машина, работающая по замкнутому термодинамическому циклу, в которой циклические процессы сжатия и расширения происходят при различных уровнях температур, а управление потоком рабочего тела осуществляется путем изменения его объема. Этот принцип положен в основу преобразования теплоты в работу или, наоборот, работы в теплоту для большого семейства машин Стирлинга, различающихся по своим функциям, характеристикам и конструктивным схемам. Эти двигатели могут быть роторными и поршневыми с конструктивной схемой различной степени сложности. Указанные машины способны работать как двигатели, тепловые насосы, холодильные установки и компрессоры. Известно, что в настоящее время многие крупные мировые корпорации и научные институты проводят исследования в этой области. В нашей стране в этом направлении активно работают с 60-х гг. прошлого века. Вместе с тем отсутствуют методики нестационарного численного расчета газодинамических процессов в двигателе Стирлинга, которые позволили бы оптимизировать процесс, повысить его эффективность. В работе [1] при- 
водятся сравнительные характеристики различных типов двигателей (табл.1).

Таблица 1

Сравнительная характеристика типов двигателей [1]

\begin{tabular}{|l|c|c|c|c|c|}
\hline \multicolumn{1}{|c|}{ Тип двигателя } & $\begin{array}{c}\text { Двигатель } \\
\text { Стирлинга }\end{array}$ & $\begin{array}{c}\text { Бензиновый } \\
\text { двигатель }\end{array}$ & $\begin{array}{c}\text { Дизельный } \\
\text { двигатель }\end{array}$ & $\begin{array}{c}\text { Газотурбинный } \\
\text { двигатель }\end{array}$ & $\begin{array}{c}\text { Топливные } \\
\text { элементы }\end{array}$ \\
\hline $\begin{array}{l}\text { Стоимость за } \\
1 \text { кВт, тыс. долл. } \\
\text { США }\end{array}$ & $1-3,5$ & $0,4-1,5$ & $0,6-2,5$ & $3-3,5$ & $6-12$ \\
\hline КПд & $24-43$ & $15-18$ & $15-32$ & $20-30$ & $22-50$ \\
\hline Вид топлива & $\begin{array}{c}\text { Многотоп- } \\
\text { ливный }\end{array}$ & $\begin{array}{c}\text { Бензин } \\
\text { АИ-92 }\end{array}$ & $\begin{array}{c}\text { Дизельное } \\
\text { топливо }\end{array}$ & $\begin{array}{c}\text { Природный } \\
\text { газ }\end{array}$ & Водород \\
\hline Замена масла & Отсутствует & 100 ч & 100 ч & Отсутствует & Отсутствует \\
\hline $\begin{array}{l}\text { Уровень шума, } \\
\text { дБ }\end{array}$ & $\begin{array}{c}60-65 \\
(1 \text { м })\end{array}$ & $\begin{array}{c}70-77 \\
(7-15 ~ м)\end{array}$ & $\begin{array}{c}80-94 \\
(7-15 \text { м })\end{array}$ & $\begin{array}{c}\text { Свыше } \\
90-100\end{array}$ & 60 (1 м) \\
\hline $\begin{array}{l}\text { Моторесурс, } \\
\text { тыс. ч }\end{array}$ & до 80 & до 5 & до 25 & до 30 & от 2 до 6 \\
\hline
\end{tabular}

Из табл. 1 видно, что двигатель Стирлинга обладает рядом преимуществ, и требуются дополнительные исследования в этом направлении.

\section{Физическая модель}

Сформулирована следующая физическая постановка задачи:

- конструкция полагается трехмерной $(x, y, z)$;

- необходимо исследовать нестационарные колебания газа при вынужденных колебаниях конструкции, так как периоды колебаний поршней и газа имеют одинаковый порядок;

- в качестве рабочего тела выбран совершенный газ;

- используется численно-аналитический подход для моделирования колебаний в динамической системе газ - конструкция. Колебания вала описываются аналитически гармоническим законом, они вызывают колебания в газе, процессы в котором описываются численно;

- процессы рассматриваются трехмерными динамическими. Исследуется одно колебание, так как в двигателе Стирлинга установление рабочего процесса происходит за малую часть периода; 
- расчетная схема включает в себя два подвижных тела, которые имитируют поршни; поршни двигаются по гармоническому закону. Принимается, что поршни могут совершать как зависимые, так и независимые колебания одинаковой частоты, но отличающиеся по фазе, которая не изменяется при работе [2-6];

- расчеты проводятся как с учетом подвода тепла, так и без него. Подвод тепла моделируется температурой стенки под большим поршнем;

- при этом фиксируется распределение газодинамических сил на поршнях. Особый интерес представляет интервал сдвига фаз вблизи $90^{\circ}$, поэтому сдвиг фаз между колебаниями поршней в расчетах принимается равным следующим фиксированным значениям: 90, 92, $88^{\circ}$ [7-15].

Расчетная схема двигателя Стирлинга приводится на рис. 1.

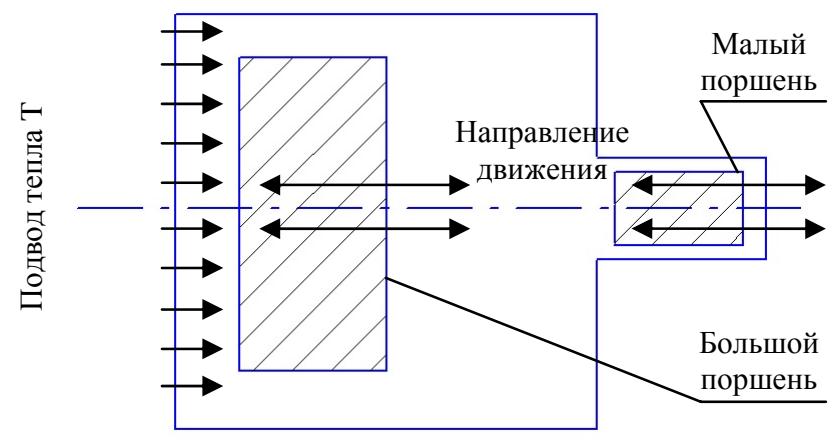

Рис. 1. Расчетная схема

\section{Математическая модель}

Математическая модель включает в себя следующие соотношения:

- закон сохранения массы

$$
\frac{\partial \rho}{\partial t}+\nabla(\rho \vec{v})=0
$$

- закон сохранения импульса

$$
\frac{\partial \rho \vec{v}}{\partial t}+\nabla(\rho \vec{v} \otimes \vec{v})=-\nabla p+\nabla\left(\left(\mu+\mu_{t}\right)\left(\nabla \vec{v}+(\nabla \vec{v})^{T}\right)\right) ;
$$


- закон сохранения энергии

$$
\frac{\partial(\rho H)}{\partial t}+\nabla(\rho \vec{v} H)=\frac{\partial p}{\partial t}+\nabla\left(\left(\frac{\lambda}{c_{p}}+\frac{\mu_{t}}{\operatorname{Pr}_{t}}\right) \nabla H\right)
$$

где $t$ - время; $\rho$ - плотность; $v$ - скорость; $p$ - давление; $\mu$ - динамическая вязкость; $\mu_{t}$ - турбулентная динамическая вязкость; $H$ - энтальпия; $c_{p}$ - теплоемкость; $\lambda$ - коэффициент теплопроводности; $\operatorname{Pr}_{p}-$ турбулентное число Прандтля.

Используется $k-\varepsilon$-модель турбулентности. Вводятся следующие соотношения:

$$
\begin{gathered}
\frac{\partial(\rho k)}{\partial t}+\nabla(\rho \vec{v} k)=\nabla\left(\left(\mu+\frac{\mu_{t}}{\sigma_{k}}\right) \nabla k\right)+\mu_{t} G-\rho \varepsilon \\
\frac{\partial(\rho \varepsilon)}{\partial t}+\nabla(\rho \vec{v} \varepsilon)=\nabla\left(\left(\mu+\frac{\mu_{t}}{\sigma_{\varepsilon}}\right) \nabla \varepsilon\right)+C_{1} \frac{\varepsilon}{k} \mu_{t} G-C_{2} f_{1} \rho \frac{\varepsilon^{2}}{k},
\end{gathered}
$$

где $G=D_{i j} \frac{\partial v_{i}}{\partial x_{i}}, \quad D_{i j}=S_{i j}-\frac{2}{3}\left(\nabla \cdot \vec{v}+\frac{\rho k}{\mu_{t}}\right) \delta_{i j}, \quad S_{i j}=\frac{\partial v_{i}}{\partial x_{j}}+\frac{\partial v_{j}}{\partial x_{i}} ; \quad \sigma_{k}=1 ;$ $\sigma_{\varepsilon}=1,3 ; C_{1}=1,44 ; C_{2}=1,92 ; f_{1}=1 ; k-$ турбулентная энергия; $\varepsilon-$ скорость диссипации турбулентной энергии; $G$ - определяет скорость генерации турбулентной энергии; $S_{i j}-$ удвоенный тензор скоростей деформации.

Уравнение турбулентной вязкости вычисляется по формуле Колмогорова-Прандтля:

$$
\mu_{t}=C_{\mu} \rho \frac{k^{2}}{\varepsilon}
$$

где $C_{\mu}=0,09-$ константа;

- уравнение состояния

$$
p=\rho R T
$$

где $R$ - газовая постоянная.

Математическая модель замыкается начальными и граничными условиями. Начальные значения температуры и давления следующие: 
$T_{\text {нач }}=20{ }^{\circ} \mathrm{C}, p_{\text {нач }}=0,1 \mathrm{MПа,} \mathrm{колебательное} \mathrm{движение} \mathrm{стенок} \mathrm{поршней}$ с заданной частотой и амплитудой было реализовано через условие Mesh deformation и законом движения $A=A_{0} \cdot \sin (2 \pi / T \cdot t)$.

Расчетная схема позволяет проводить вычислительные эксперименты, снизив требования к вычислительным ресурсам. Данные расчеты проводились на одном узле высокопроизводительного вычислительного комплекса ПНИПУ, включающем в себя 16 ядер процессоров Intel Xeon E5-2680, объем оперативной памяти 128 Гбайт/узел, операционную систему Windows HPC Server 2008.

\section{Результаты расчета}

Полученые результаты отображены на рис. 2. В левой части рисунка $2(2, a, в, \partial, ж, \kappa, м)$ приводятся зависимости для большого поршня (БП) (см. рис. 1). В правой части рис. 2 (2, б, г, e, з, л, н) приводятся зависимости для малого поршня (МП). На всех графиках по вертикальной оси откладываются величина результирующей газодинамической силы, действующей на соответствующий поршень, и его перемещения. Каждая пара рисунков: $2, a-2, \sigma ; 2, в-2,2 ; 2, \partial-2, e ; 2, ж-$ 2,$3 ; 2, \kappa-2, \pi ; 2, \mu-2, \mu-$ соответствует определенным значениям угла свига фаз и условиям подвода тепла.

При сдвиге фаз $88^{\circ}$ колебания газодинамической силы и поршней происходят в противофазе без подвода тепла, а при подводе тепла в противофазе движется только правый поршень.

При сдвиге фаз $90^{\circ}$ для большого поршня колебания газодинамических сил и перемещений поршня синфазны, а для малого поршня находятся в противофазе.

При сдвиге фаз $92^{\circ}$ без подвода тепла колебания правого и левого поршней и соответсвующих газодинамических сил синфазны, а при подводе тепла колебания происходят в противофазе.

В табл. 2 представлены сдвиги фаз колебаний в конструкции (заданы) и в газе (получены расчетным путем). 

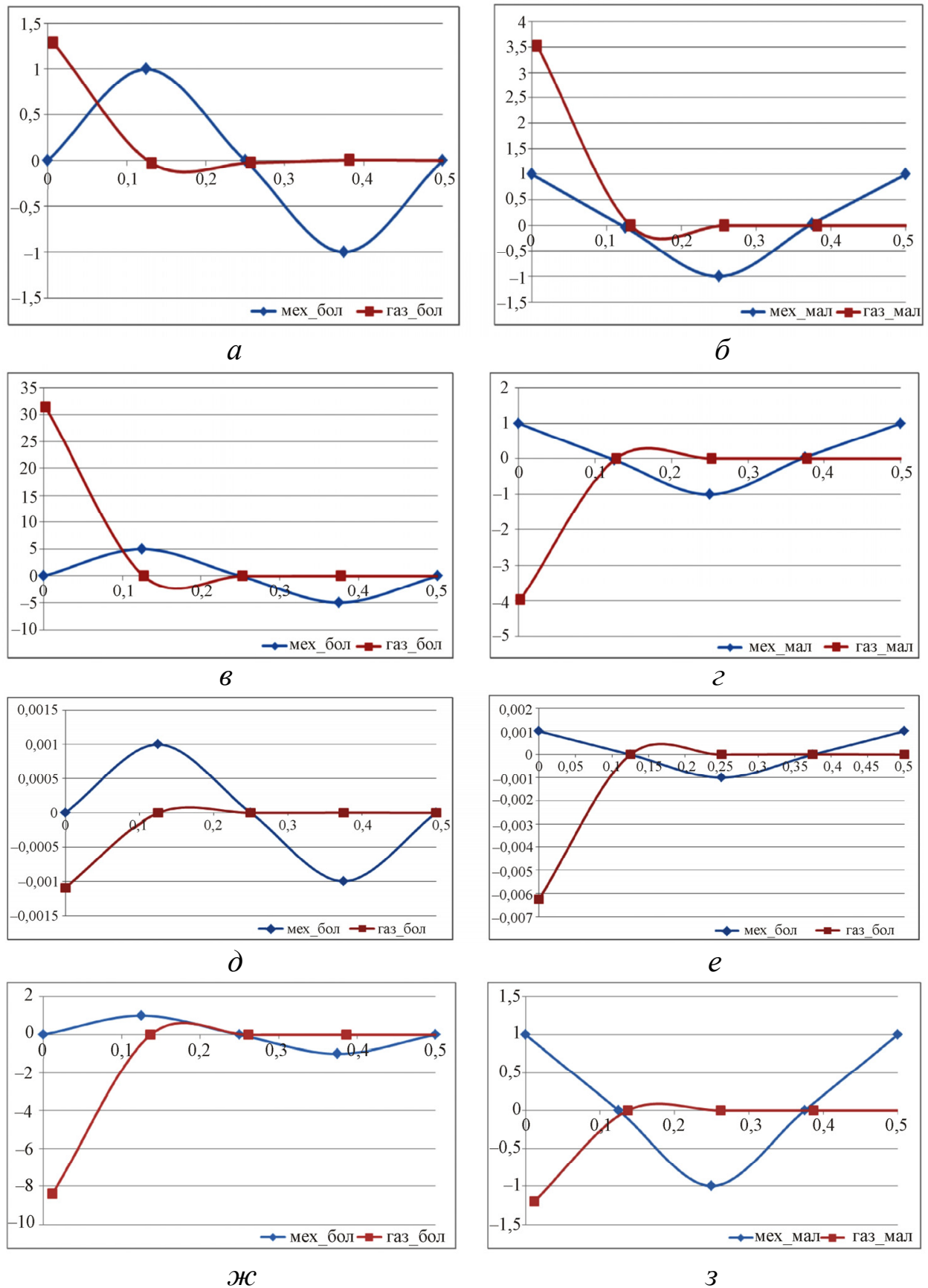

Рис. 2. Зависимость газодинамических сил от времени при следующих значениях сдвига фаз колебаний поршней: $a-\varphi=88^{\circ}, T=100{ }^{\circ} \mathrm{C}$ (БП); $\sigma-\varphi=88^{\circ}, T=100{ }^{\circ} \mathrm{C}$ (МП); $в-\varphi=88^{\circ}, T=T_{\text {нач }}\left(\right.$ БП); $2-\varphi=88^{\circ}, T=T_{\text {нач }}(\mathrm{M \Pi}) ; \partial-\varphi=90^{\circ}, T=100{ }^{\circ} \mathrm{C}(\mathrm{M \Pi})$; $e-\varphi=90^{\circ}, T=100^{\circ} \mathrm{C}\left(\right.$ БП); $\nsim-\varphi=90^{\circ}, T=T_{\text {нач }}(\mathrm{MП}) ; 3-\varphi=90^{\circ}, T=T_{\text {нач }}($ БП $)$

(Окончание см. на с. 109) 

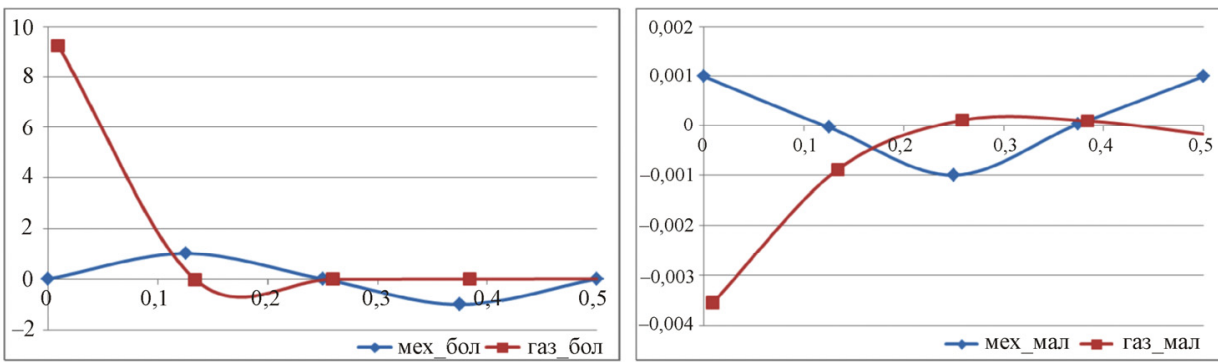

$\kappa$

$\pi$
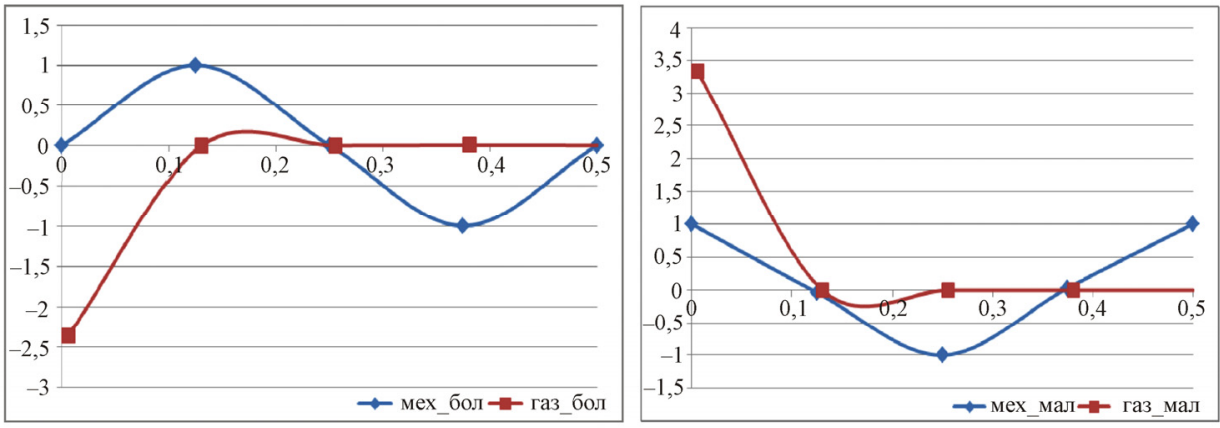

$\mathcal{M}$

$H$

Рис. 2. Окончание: $\kappa-\varphi=92^{\circ}, T=100{ }^{\circ} \mathrm{C}(\mathrm{MП}) ; \pi-\varphi=92^{\circ}, T=100{ }^{\circ} \mathrm{C}$ (БП); $\mathcal{M}-\varphi=92^{\circ}, T=T_{\text {нач }}\left(\mathrm{MП);} \mu-\varphi=92^{\circ}, T=T_{\text {нач }}(\right.$ БП) (Начало см. на с. 108 )

Таблица 2

Сдвиги фаз колебаний в конструкции и в газе

\begin{tabular}{|c|c|c|c|c|c|}
\hline \multirow[t]{2}{*}{$\begin{array}{l}\text { № } \\
\Pi / \Pi\end{array}$} & \multirow{2}{*}{$\begin{array}{c}\text { Конструкция. } \\
\text { Сдвиг фаз } \\
\text { колебаний } \\
\text { поршней } \varphi_{1}, \\
\text { град }\end{array}$} & \multicolumn{2}{|c|}{$\begin{array}{c}\text { Сдвиг фаз колебаний } \\
\text { между поршнем } \\
\text { и действующей на него } \\
\text { газодинамической силой }\end{array}$} & \multirow{2}{*}{$\begin{array}{c}\text { Газодинамика. } \\
\text { Сдвиг фаз колеба- } \\
\text { ний газодинамиче- } \\
\text { ских сил, действую- } \\
\text { щих на поршни, } \varphi_{4}\end{array}$} & \multirow[t]{2}{*}{$\begin{array}{c}\text { Подвод } \\
\text { тепла }\end{array}$} \\
\hline & & $\begin{array}{c}\text { Правый } \\
\text { поршень } \varphi_{2}\end{array}$ & $\begin{array}{c}\text { Левый } \\
\text { поршень } \varphi_{3}\end{array}$ & & \\
\hline 1 & 88 & $\pi$ & $\pi$ & $-\pi / 24$ & - \\
\hline 2 & 88 & $\pi$ & 0 & $-\pi / 24$ & + \\
\hline 3 & 90 & 0 & $\pi$ & $-\pi / 24$ & - \\
\hline 4 & 90 & 0 & $\pi$ & $-\pi / 24$ & + \\
\hline 5 & 92 & 0 & 0 & $-\pi / 24$ & - \\
\hline 6 & 92 & $\pi$ & $\pi$ & $-\pi / 4$ & + \\
\hline
\end{tabular}

Можно сказать, что при заданной фазе колебаний поршней, равной 90 и $88^{\circ}$, газодинамические силы на поршнях имеют небольшой 
сдвиг фаз, причем угол сдвига фаз имеет отрицательное значение. При заданном сдвиге фаз $92^{\circ}$ газодинамические силы на поршнях имеют сдвиг фаз при подводе тепла, равный $-45^{\circ}$, а при холодной поверхности сдвиг фаз мал.

\section{Заключение}

1. Предложена новая расчетная схема для численного моделирования газодинамических процессов в двигателе Стирлинга, которая позволяет снизить требования к вычислительным ресурсам.

2. Обнаружено, что сдвиг фаз колебаний между поршнями влияет на соответствие колебаний поршней и газодинамических сил.

3. Анализ результатов позволяет предположить, что для эффективной работы двигателя Стирлинга необходимо и достаточно обеспечить $\varphi_{1}=\varphi_{2}=\varphi_{3}=0$.

4. Выявлены зависимости колебаний газодинамической силы, действующей на правый поршень от перемещений поршней, сдвига фаз колебаний, наличия подвода тепла, а также колебаний газодинамической силы, действующей на левый поршень от перемещений поршней, сдвига фаз колебаний, наличия подвода тепла.

При увеличении сдвига фаз между поршнями $\left(\varphi_{1}\right)$ сдвиг фаз колебаний между поршнем и действующей на него газодинамической силой $\left(\varphi_{2}, \varphi_{2}\right)$ увеличивается.

5. Выявлены зависимости сдвига фаз колебаний между поршнем и действующей на него газодинамической силой $\left(\varphi_{2}, \varphi_{3}\right)$ от сдвига фаз колебаний поршней $\left(\varphi_{1}\right)$.

При увеличении сдвига фаз между поршнями $\left(\varphi_{1}\right)$ сдвиг фаз колебаний между поршнем и действующей на него газодинамической силой $\left(\varphi_{2}, \varphi_{3}\right)$ равен 0 или $\pi$, в зависимости от того, есть ли источник тепла.

Работа выполнена при финансовой поддержке гранта Российского научного фонда (проект № 14-19-00877).

\section{Библиографический список}

1. Кириллов Н.Г., Передерий С.Э. Перспективы создания автономных систем комбинированного производства электрической и тепловой энергии на основе сжигания биотоплива в двигателях Стирлинга 
[Электронный ресурc]. - URL: http://www.infobio.ru/sites/default/files/ Kirillov-Perederi.pdf (дата обращения: 20.01.2016).

2. Трехмерное моделирование флаттера лопаток компрессоров современных ГТД / В.В. Веденеев, М.Е. Колотников, П.В. Макаров, В.В. Фирсанов // Вестник Самар. гос. аэрокосм. ун-та им. акад. С.П. Королёва (нац. исслед. ун-та). - 2011. - Вып. 3-1. - С. 47-55.

3. Mekhonoshina E.V., Modorskii V.Y. On a phase-shift of waves at the medium interface // Computer Optics. - 2015. - Vol. 39, iss. 3. P. 385-391.

4. Mekhonoshina E.V., Modorskii V.Ya., Petrov V.Yu. Numeric simulation of the interaction between subsonic // Source of the Document CEUR Workshop Proceedings. Conference "Information Technology and Nanotechnology (ITNT - 2015). Mathematical Modeling". - P. 211-218.

5. Numerical modeling of flow mixing and cooling processes in a perforated exhaust duct / A.N. Ponik, V.Ya. Modorskii, A.V. Kozlova, Yu.V. Sokolkin // Russian Aeronautics (Iz VUZ). - 2014. - Vol. 57, № 2. P. 181-186.

6. Modorskii V.Y., Petrov V.Y. Aeroelastic deformation of a solidpropellant rocket engine during supercharging // Russian Aeronautics (Iz VUZ). - 2011. - Vol. 54, № 3. - P. 272-275.

7. Петров В.Ю., Модорский В.Я., Бутымова Л.Н. Численное моделирование влияния колебаний лопаток модельного компрессора на систему «газ - конструкция» // Науч.-техн. вестник Поволжья. - 2015. № 5. - С. 161-163.

8. Модорский В.Я., Бутымова Л.Н. Исследование колебательных процессов на резонансных режимах в модельной установке // Науч.техн. вестник Поволжья. - 2013. - № 6. - С. 193-196.

9. Соколкин Ю.В., Модорский В.Я., Бутымова Л.Н. Разработка экспериментальной установки и исследование влияния материала корпуса на резонансные частоты в системе «газ - конструкция» // Науч.техн. вестник Поволжья. - 2013. - № 6. - С. 197-200.

10. Инженерная методика определения настроек газохода переменного сечения для отвода и охлаждения горячих газов энергетических установок / В.Ю. Петров, В.Я. Модорский, А.Н. Поник, А.В. Козлова // Науч.-техн. вестник Поволжья. - 2012. - № 2. - С. 216-219.

11. Численная оценка напряженно-деформированного состояния лопатки компрессора в аэроупругой постановке / В.Ю. Петров, 
В.Я. Модорский, А.И. Плотников, Е.В. Мехоношина // Науч.-техн. вестник Поволжья. - 2015. - № 5. - С. 235-238.

12. Петров В.Ю., Модорский В.Я., Шмаков А.Ф. Численное моделирование газодинамических процессов и процессов деформирования в компрессоре модельного стенда газоперекачивающего агрегата // Науч.-техн. вестник Поволжья. - 2015. - № 5. - С. 318-320.

13. Shmakov A.F., Modorskii V.Ya. Energy conservation in cooling systems at metallurgical plants // Metallurgist. - 2016. - Vol. 59, iss. 9. Р. 882-886.

14. Модорский В.Я., Соколкин Ю.В. Газоупругие процессы в энергетических установках. - М.: Наука, 2007. - 176 с.

15. Бутымова Л.Н., Модорский В.Я. Исследование газодинамического потока и конструкции в модельной экспериментальной установке // Вестник ЮУрГУ. Сер.: Вычислительная математика и информатика. - 2014. - Т. 3, № 2. - С. 92-100.

\section{References}

1. Kirillov N.G., Perederiy S.E. Perspektivy sozdaniya avtonomnykh sistem kombinirovannogo proizvodstva elektricheskoy i teplovoy energii na osnove szhiganiya biotopliva $\mathrm{v}$ dvigatelyakh Stirlinga [Prospects of creation of independent systems for generation of electric and heat energy based on biofuel combustion in Stirling engines], available at: http://www.infobio.ru/sites/default/files/Kirillov-Perederi.pdf (accessed 20 January 2016).

2. Vedeneev V.V., Kolotnikov M.E., Makarov P.V., Firsanov V.V. Trekhmernoe modelirovanie flattera lopatok kompressorov sovremennykh GTD [Three-dimensional simulation of compressor blade flutter of the modern gas-turbine engine]. Vestnik Samarskogo gosudarstvennogo aerokosmicheskogo universiteta imeni akademika S.P. Koroleva (natsionalnogo issledovatelskogo universiteta), 2011, iss. 3-1, pp. 47-55.

3. Mekhonoshina E.V., Modorskii V.Y. On a phase-shift of waves at the medium interface. Computer Optics, 2015, vol. 39, iss. 3, pp. 385-391.

4. Mekhonoshina E.V., Modorskii V.Ya., Petrov V.Yu. Numeric simulation of the interaction between subsonic. Source of the Document CEUR Workshop Proceedings. Conference "Information Technology and Nanotechnology (ITNT - 2015). Mathematical Modeling”, pp. 211-218. 
5. Ponik A.N., Modorskii V.Ya., Kozlova A.V., Sokolkin Yu.V. Numerical modeling of flow mixing and cooling processes in a perforated exhaust duct. Russian Aeronautics (Iz VUZ), 2014, vol. 57, no. 2, p. 181-186.

6. Modorskii V.Y., Petrov V.Y. Aeroelastic deformation of a solidpropellant rocket engine during supercharging // Russian Aeronautics (Iz VUZ), 2011, vol. 54, no. 3, pp. 272-275.

7. Petrov V.Yu., Modorskiy V.Ya., Butymova L.N. Chislennoe modelirovanie vliyaniya kolebaniy lopatok modelnogo kompressora na sistemu "gaz - konstruktsiya" [Numerical simulation of influence of model compressor blade oscillations on "gas-design" system]. Nauchnotekhnicheskiy vestnik Povolzhya, 2015, no. 5, pp. 161-163.

8. Modorskiy V.Ya., Butymova L.N. Issledovanie kolebatelnykh protsessov na rezonansnykh rezhimakh $\mathrm{v}$ modelnoy ustanovke [Research of oscillation processes at resonance modes in model set]. Nauchnotekhnicheskiy vestnik Povolzhya, 2013, no. 6, pp. 193-196.

9. Sokolkin Yu.V., Modorskiy V.Ya., Butymova L.N. Razrabotka eksperimentalnoy ustanovki i issledovanie vliyaniya materiala korpusa na rezonansnye chastoty v sisteme "gaz - konstruktsiya" [Development of electric installation and research of influence of case material on resonance frequencies in "gas-design" system]. Nauchno-tekhnicheskiy vestnik Povolzhya, 2013, no. 6, pp. 197-200.

10. Petrov V.Yu., Modorskiy V.Ya., Ponik A.N., Kozlova A.V. Inzhenernaya metodika opredeleniya nastroek gazokhoda peremennogo secheniya dlya otvoda i okhlazhdeniya goryachikh gazov energeticheskikh ustanovok [Engineering technique for determination of parameters of variable cross-section gas flue to withdraw and cool the gases of electric installations]. Nauchno-tekhnicheskiy vestnik Povolzhya, 2012, no. 2, pp. 216-219.

11. Petrov V.Yu., Modorskiy V.Ya., Plotnikov A.I., Mekhonoshina E.V. Chislennaya otsenka napryazhenno-deformirovannogo sostoyaniya lopatki kompressora $\mathrm{v}$ aerouprugoy postanovke [Numerical evaluation of stress strain state of compressor blade at aeroelastic state]. Nauchnotekhnicheskiy vestnik Povolzhya, 2015, no. 5, pp. 235-238.

12. Petrov V.Yu., Modorskiy V.Ya., Shmakov A.F. Chislennoe modelirovanie gazodinamicheskikh protsessov i protsessov deformirovaniya $\mathrm{v}$ kompressore modelnogo stenda gazoperekachivayushchego agregata 
[Numerical simulation of gas-dynamic processes and deformation processes in compressor of modelling test branch of gascompressor unit]. Nauchnotekhnicheskiy vestnik Povolzhya, 2015, no. 5, pp. 318-320.

13. Shmakov A.F., Modorskii V.Ya. Energy conservation in cooling systems at metallurgical plants. Metallurgist, 2016, vol. 59, iss. 9, pp. 882-886.

14. Modorskiy V.Ya., Sokolkin Yu.V. Gazouprugie protsessy v energeticheskikh ustanovkakh [Gas pressure processes in power plants]. Moscow: Nauka, 2007. 176 p.

15. Butymova L.N., Modorskiy V.Ya. Issledovanie gazodinamicheskogo potoka i konstruktsii v modelnoy eksperimentalnoy ustanovke [Study of gasdynamic flow and design in model test facility]. Vestnik YuzhnoUralskogo gosudarstvennogo univesiteta. Vychislitelnaya matematika i informatika, 2014, vol. 3, no. 2, pp. 92-100.

\section{Об авторах}

Бутымова Лилия Николаевна (Пермь, Россия) - инженер Центра высокопроизводительных вычислительных систем ФГБОУ ВПО ПНИПУ (614990, г. Пермь, Комсомольский пр., д. 29, e-mail: liliya85@ gmail.com).

Модорский Владимир Яковлевич (Пермь, Россия) - доктор технических наук, профессор кафедры «Механика композиционных материалов и конструкций» ФГБОУ ВПО ПНИПУ (614990, г. Пермь, Комсомольский пр., д. 29, e-mail: modorsky@pstu.ru).

Николай Алексеевич Шевелев (Пермь, Россия) - доктор технических наук, профессор, ФГБОУ ВПО ПНИПУ (614990, г. Пермь, Комсомольский пр., д. 29, e-mail: modorsky@pstu.ru).

\section{About the authors}

Liliya N. Butymova (Perm, Russian Federation) - Engineer, Center for High Performance Computing Systems, Perm National Research Polytechnic University (29, Komsomolsky av., Perm, 614990, Russian Federation, e-mail: liliya85@gmail.com).

Vladimir Ya. Modorskiy (Perm, Russian Federation) - Doctor of Technical Sciences, Professor, Mechanics of Composite Materials and Constructions Department, Perm National Research Polytechnic University 
(29, Komsomolsky av., Perm, 614990, Russian Federation, e-mail: modorsky@pstu.ru).

Nikolay A. Shevelev (Perm, Russian Federation) - Doctor of Technical Sciences, Professor, Perm National Research Polytechnic University (29, Komsomolsky av., Perm, 614990, Russian Federation, e-mail: n.shevelev@pstu.ru).

Получено 29.01.2016 